\title{
THE EFFECTS OF LOW-LEVEL LASER ON MUSCLE DAMAGE CAUSED BY
}

\section{Bothrops neuwiedi VENOM}

\author{
DOURADO DM (1), MATIAS R (1), ALMEIDA MF (1), DE PAULA KR(1), VIEIRA \\ RP (3), OLIVEIRA LVF (2), CARVALHO PTC (1)
}

(1) Laboratory of Experimental Histopathology, University for the Development of the State and of the Region of Pantanal, UNIDERP, Campo Grande, Mato Grosso do Sul State, Brazil; (2) Masters Program in Rehabilitation Sciences, Nove de Julho University, UNINOVE, São Paulo, São Paulo State, Brazil; (3) Department of Pathology and Physical Therapy, School of Medicine, University of São Paulo, São Paulo, São Paulo State, Brazil.

\begin{abstract}
The present study aimed to assess the effects of low-level laser (660 $\mathrm{nm}$ ) on myonecrosis caused by the insertion of Bothrops neuwiedi venom in the gastrocnemius muscle of rats. Male Wistar rats were divided into three groups $(n=$ 24 each): Group S (0.9\% saline solution); Group V (venom) and Group VLLL (venom plus low-level laser). These categories were subdivided into four additional groups ( $\mathrm{n}$ $=6$ ) based on the euthanasia timing ( 3 hours, 24 hours, 3 days and 7 days). The groups $V$ and VLLL were inoculated with $100 \mu \mathrm{L}$ of concentrated venom $(40 \mu \mathrm{g} / \mathrm{mL})$ in the gastrocnemius muscle. The muscle was irradiated using a gallium-aluminumarsenide laser (GaAlAs) at $35 \mathrm{~mW}$ power and $4 \mathrm{~J} / \mathrm{cm}^{2}$ energy density for 3 hours, 24 hours, 3 days or 7 days after venom inoculation. To evaluate the myotoxic activity of the venom, CK activity was measured and the muscle was histologically analyzed. The low-level laser reduced venom-induced CK activity in the groups euthanized at 3 hours, 24 hours and 3 days $(p<0.0001)$. Histological analysis revealed that low-level laser reduced neutrophilic inflammation as well as myofibrillar edema, hemorrhage and myonecrosis following $B$. neuwiedi envenomation. These results suggest that low-level laser can be useful as an adjunct therapy following $B$. neuwiedi envenomation.
\end{abstract}

KEY WORDS: myonecrosis, B. neuwiedi venom, low-level laser.

CONFLICTS OF INTEREST: There is no conflict.

\section{CORRESPONDENCE TO:}

DOROTY MESQUITA DOURADO, Rua Ceara, 333, Bairro Miguel Couto, Campo Grande, MS, 79032423, Brazil. Phone: +55 67 33488366. Email: ccfi@uniderp.br. 


\section{INTRODUCTION}

The Bothrops neuwiedi (Neuwied's lancehead) species complex consists of a variety of subspecies widely distributed throughout South America (1). In general, the prevalence of Bothrops ophidian accidents in Brazil is approximately $85 \%$ when compared with other species (24). Bothrops neuwiedi venom causes fibrinolytic, hemorrhagic and anticoagulant effects $(9,12)$. The main local actions are tissue necrosis and endothelial injury, while systemic effects include release of inflammatory and vasoactive mediators $(1,17,22)$. Envenomation by Bothrops snakes results in serious wounds, muscular necrosis, pain, edema, ecchymosis, abscess and hemodynamic changes (24).

The affected area exhibits ischemia as a consequence of serious vascular lesions and profuse bleeding. Hemorrhage is caused by the action of metalloproteases, which damages the collagen and other capillary vessel components of the basal lamina. As a result, the capillary vessels are broken, causing ecchymosis. These dysfunctions and local wounds are specific clinical symptoms of $B$. neuwiedi envenomation (17). Several myotoxins have been isolated from Bothrops snake venoms and their effects appear to be similar to other toxins found in crotalic venoms, such as Crotalus durissus terrificus (23). Intramuscular injection of Bothrops myotoxins leads to a rapid series of drastic degenerative events, probably initiated at the plasma membrane level, that culminate in selective skeletal muscle necrosis. Muscle damage, cytolysis and liposome disruption occur under conditions in which phospholipase A2 activity is inhibited, while enzymatic activity may enhance the myotoxin action (9).

A large number of researchers have been looking for new therapeutic methods to repair or at least minimize the damage caused by Bothrops snake venom to the muscles $(3,5,10)$. Low-level laser therapy can be considered an alternative if we consider its sequelae on different types of biological tissues - including its trophicregenerative, anti-inflammatory and analgesic effects - which have been demonstrated in both in vitro and in vivo studies $(2,4,6-8,11,18,19,21,24-26)$.

The present study aimed to assess the effects of low-level laser $(660 \mathrm{~nm})$ on myonecrosis caused by the injection of $B$. neuwiedi venom into the gastrocnemius muscle of Wistar rats. 


\section{MATERIALS AND METHODS}

\section{Venom}

Natural venom was extracted from $B$. neuwiedi snakes that were captured in the Pantanal region on Mato Grosso do Sul State, Brazil, and kept at the Zoology Laboratory of the University for the Development of the State and of the Region of Pantanal, UNIDERP, Campo Grande. This extracted venom was lyophilized and stored at $-20^{\circ} \mathrm{C}$.

\section{Animals}

Seventy-two adult male Wistar rats (Rattus norvegicus albinus), weighing 200 to 300 $\mathrm{g}$, obtained from the central animal house at UNIDERP, were kept in 12 cages (six animals per cage) under controlled lighting and temperature conditions, with standard food and water available ad libitum.

All the experimental procedures were carried out according to the rules of the Brazilian College for Animal Experimentation (COBEA) and approved by the UNIDERP Research Ethics Committee, protocol number 185/2006/CEP.

\section{Experimental Groups}

The 72 animals were randomly divided into three experimental groups $(n=24)$ : Group S (sterile $0.9 \%$ saline solution - $0.1 \mathrm{~mL} / 100 \mathrm{~g}$ ); Group V (natural venom solution) and Group VLLL (natural venom treated with low-level laser). These were further subdivided into four additional groups $(n=6)$ based on the euthanasia timing (3 hours, 24 hours, 3 days and 7 days).

\section{Venom Inoculation}

The animals were anesthetized with sodium pentobarbital (40-70 mg/kg, IM). A total of $100 \mu \mathrm{L}$ of the venom solution at the concentration of $40 \mu \mathrm{g}$ was inoculated intramuscularly in the gastrocnemius muscle of the groups venom (V) and venomwith-low-level-laser-application (VLLL). The control animals were inoculated with a sterile $0.9 \%$ saline solution.

\section{Application of Low-level Laser}

A gallium-aluminum-arsenide (GaAIAs) laser emitter (KLD ${ }^{\circledR}$ Endophoton model) was used at $660 \mathrm{~nm}$ wavelength, $26.3 \mathrm{~mW}$ power and $0.63 \mathrm{~cm}^{2}$ beam area in continuous 
mode. The application method was transcutaneous at specific points and in contact with the skin. Energy density was $4 \mathrm{~J} / \mathrm{cm}^{2}$, power density was $0.0413 \mathrm{~W} / \mathrm{cm}^{2}$ and the length of application was 96.7 seconds, based on Amaral et al. (3), Carvalho et al. (7) and Dourado et al. (10). The average wattage of the equipment was measured before the experiment with the aid of a power-measuring device (2-watt broadband power/energy meter, 13 PEM 001/J®, Melles Griot, The Netherlands).

The gastrocnemius muscle was irradiated once a day with $4 \mathrm{~J} / \mathrm{cm}^{2}$ for seven days following the injection of the $B$. neuwiedi venom. The animals were placed in lateral decubitus position and the paw to be treated was extended and externally rotated. The laser was applied directly to the shaved skin on the right side of medial prominence of the gastrocnemius muscle.

\section{Myotoxic Activity - Creatine Kinase (CK)}

For the assessment of venom myotoxic activity, blood levels of the enzyme creatine kinase $(\mathrm{CK})$ were quantified based on the procedure described by Gutiérrez and Lomonte (12). Blood samples were collected through a heart puncture and centrifuged (Excelsa ${ }^{\circledR}$ IV Model 280R, Fanem, Brazil). Upon obtaining the sera, CK levels were immediately determined using the CK-NAC Kit ${ }^{\circ}$ (Wiener Laboratories, Argentina).

\section{Histological Analysis}

At predetermined periods of 3 hours, 24 hours, 3 days and 7 days, animals were sacrificed and muscle samples were immediately collected for histological analysis. The muscle was sectioned from the origin to the point of insertion with a scalpel blade, washed in a buffer solution and fixed in a 10\% formaldehyde solution for 24 hours. The muscle tissues were then embedded in paraffin, submitted to $5-\mu \mathrm{m}$ crosssectional cuts and stained with hematoxylin-eosin (HE).

\section{Statistical Analysis}

All data were assessed for normality using the Shapiro-Wilk test. Samples with normal distribution (parametric) were evaluated by one-way analysis of variance (ANOVA), followed by the Tukey post hoc test. Non-parametric data were evaluated using the Kruskal-Wallis test. For these procedures, the BioEstat $₫ 3.0$ statistical software was used and significance was set at $p<0.05$. 


\section{RESULTS}

At three hours, the Wistar rats inoculated with $40 \mu \mathrm{g} / \mathrm{mL}$ of raw Bothrops neuwiedi venom exhibited the following clinical signs after the venom administration: irritability, aggressiveness, muscle contraction and itching at the inoculation site as well as swelling throughout the extension of the inoculated leg for up to 12 hours. The animals inoculated with the saline solution did not exhibit any of these symptoms.

The changes observed macroscopically were confirmed by microscopic analysis. At the site of the injury, there was intensive hemorrhaging, including in the peripheral loose connective tissue that surrounds the fascicles and fibers (Figure 1). The blood vessels were congested and the muscle fibers were in different stages of degeneration, with condensed myofibrils, delta lesions, fibers with clear areas in the cytoplasm and vacuolized fibers. There was also considerable proliferation of neutrophils throughout the injured area (Figure 1).

In the animals injected with venom and irradiated with GaAIAs laser, hemorrhaging had spread throughout the whole damaged area and the erythrocytes had grouped together to form clots near the fibers. The number of fibers in degeneration was the same as in the group inoculated with venom alone. The blood vessels were highly obstructed and neutrophil infiltration appeared lower than in the group inoculated with venom alone.

In the subgroup inoculated with venom and assessed after 24 hours and 3 days, the hemorrhaging and neutrophil infiltration were greater than in all other groups. There was also an increased thickness of the fibers, various degrees of necrosis and the blood vessels continued to be congested. The presence of edema and fibers at different stages of degeneration was also found in the peripheral loose connective tissue.

At seven days, the animals injected with venom exhibited moderate mononuclear inflammatory infiltration, predominately composed of macrophages and small areas of necrosis and hemorrhaging. Moreover, the peripheral blood vessels were hyperemic. All animals treated with laser exhibited reduced injury, with the presence of myogenic cells including a large number of myoblasts and myotubes, while the muscle tissue was partially regenerated (Figure 2). 


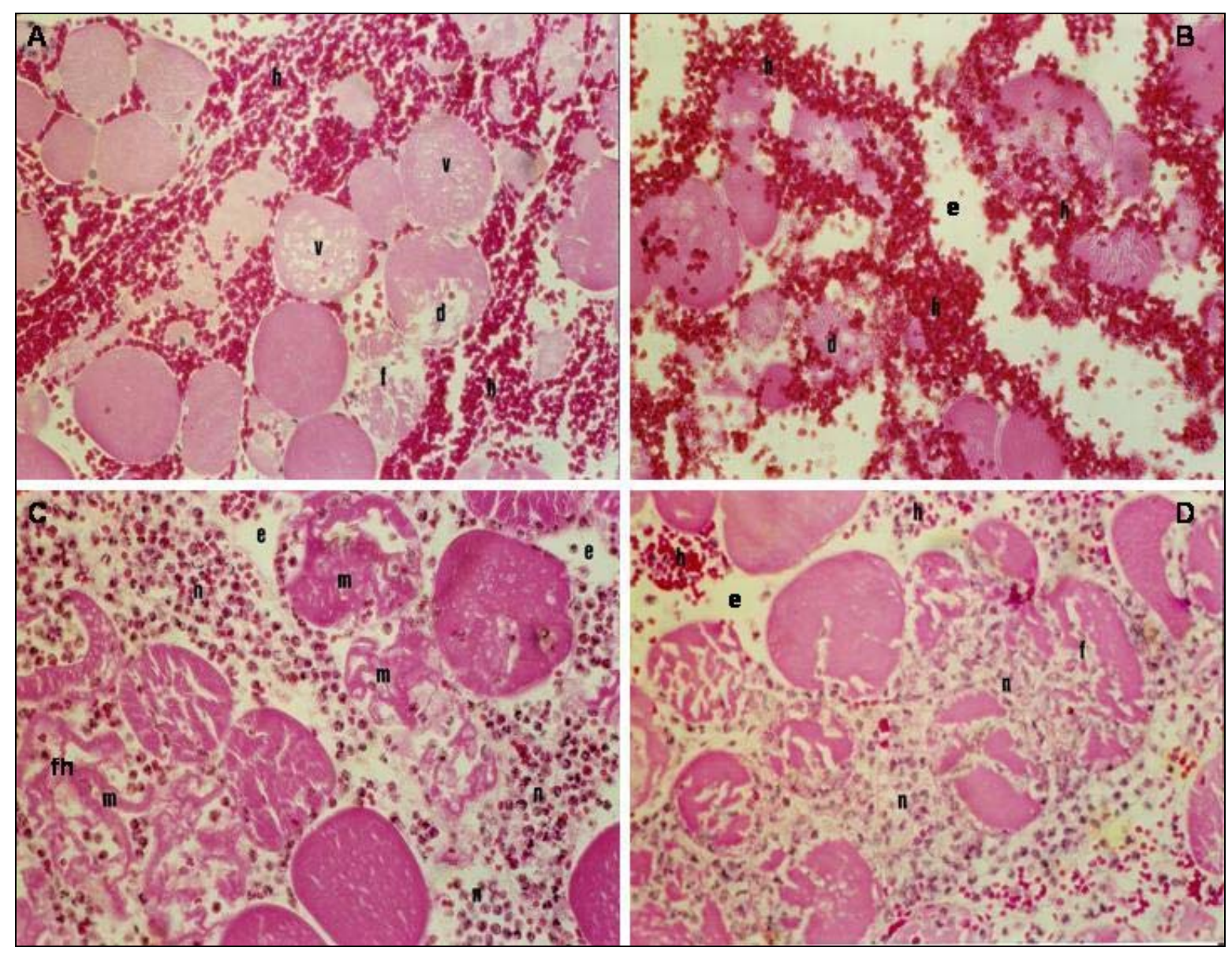

Figure 1. Cross-sections of the gastrocnemius muscle obtained at 3 hours $(\mathbf{A})$ and 24 hours (C) following injection IM of $B$. moojeni venom at a dose of $40 \mu \mathrm{g} / \mathrm{mL}$. In $A$ and C, note areas of necrosis, exsudation of neutrophils $(n)$, hemorrhaging $(h)$, densely grouped myofibrils $(\mathrm{m})$, clear areas in hypercontracted fibers $(f)$ and edema in $\mathbf{C}$. In $\mathbf{B}$ and $\mathbf{D}$, cross-sections of the gastrocnemius muscle obtained at 3 hours and 24 hours following IM injection of $B$. moojeni venom at a dose of $40 \mu \mathrm{g} / \mathrm{mL}$ and irradiated with a laser. Observe the intensive hemorrhaging $(h)$ and muscle fibers at different stages of myonecrosis in $\mathbf{B}$; and contracted fibers in $\mathbf{D}$, some exhibiting delta lesions (d), ghost cells (gc), densely grouped myofibrils (ma) and others fibers with vacuoles (v). 


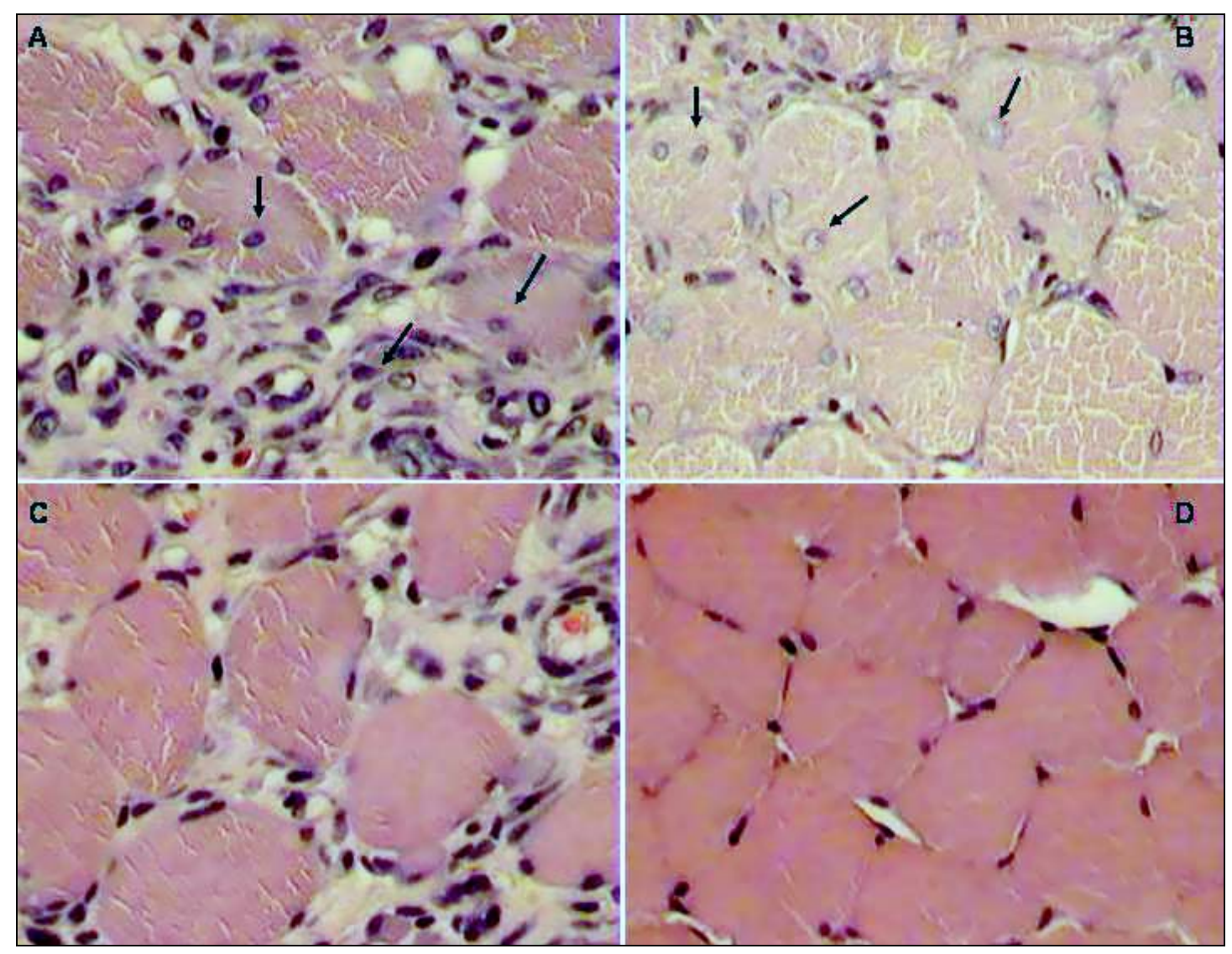

Figure 2. Cross-sections of gastrocnemius muscle ( $\mathbf{A}$ and $\mathbf{B}$ ) obtained at 3 days and 7 days following IM injection of $B$. moojeni venom at a dose of $40 \mu \mathrm{g} / \mathrm{mL}$. In A and $\mathbf{C}$, observe the appearance of young, round, corpulent fibers with a central nucleus surrounding the injured area. In $\mathbf{B}$ and $\mathbf{D}$, cross-sections of gastrocnemius muscle obtained at 3 and 7 days after IM injection of $B$. moojeni venom at a dose of 40 $\mu \mathrm{g} / \mathrm{mL}$ and laser-irradiated. Observe the regeneration of muscle tissue, culminating in D, which shows regenerated fibers and repaired tissue (HE 400x).

\section{Creatine Kinase (CK) Activity in Plasma}

Myotoxic activity was determined by measuring creatine kinase levels in plasma of the group inoculated with $40 \mu \mathrm{g}$ of venom. As Figure 3 illustrates, there was a significant increase in the release of creatine kinase in Group $V$ at all evaluation times ( 3 hours, 24 hours, 3 days and 7 days) when compared to control (Group S) and Group VLLL ( $p<0.001)$. Peak CK activity occurred three hours after venom inoculation. Figure 3 displays cross-values by CK analysis between the untreated and laser-treated subgroups: Group V (venom) versus Group VLLL (venom plus laser) for 3 hours, $p \leq 0.001 ; 24$ hours, $p \leq 0.001 ; 3$ days, $p \leq 0.001$ and 7 days, $p \geq$ 0.05 . 


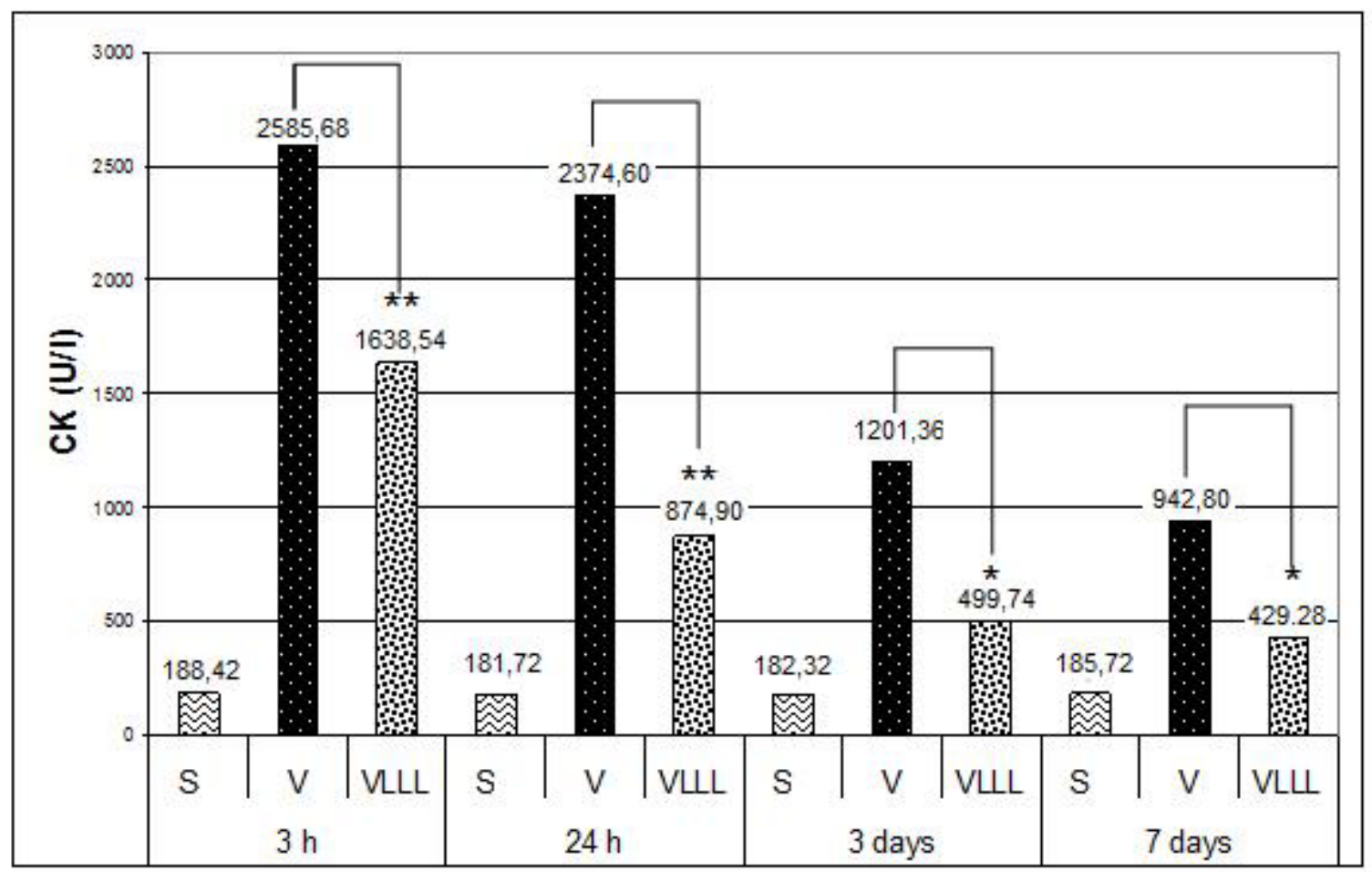

Figure 3. A bar graph representation of CK-NAC biochemical number in control, venom and GaAIAs venom-plus-laser groups. $(S=$ Sterile group, V= Venom Group, VLLL= Low-Level Laser Group)

${ }^{* *} p \leq 0.001 ;{ }^{*} p \leq 0.01$

\section{DISCUSSION}

Local tissue damage is one of the greatest problems following envenomation by snakes of the genus Bothrops; and the range of effects depends on the type of poison, the amount injected and host characteristics. Tissue damage is so intensive in many cases that it leads to serious consequences such as vascular degeneration and ischemia, which may culminate in amputation of the affected limb (17). Tissue damage is caused by the combined action of a number of venom components responsible for hemorrhaging, edema and necrosis. These toxins may be phospholipases A2 (PLA2), proteases, hemorrhagic factors and endogenous mediators released by the action of the inflammatory response $(10,11)$.

Phospholipases A2 (PLA2s) in snake venom hydrolyze phospholipids in the plasma membrane, releasing fatty acids and lysophospholipids. This leads to a catalytic process involving diverse substances, giving rise to a series of inflammatory reactions (12). Due to their catalytic function, these substances can exhibit toxic or pharmacological effects such as myonecrosis, neurotoxicity and cardiotoxicity as well 
as hemolytic, hemorrhagic, hypotensive and anticoagulant activities and inhibition of platelet aggregation (10).

The myotoxic activity of bothropic venom is a fundamental consequence of high concentrations of myotoxins. These myotoxins belong to a group of proteins with a molecular weight of approximately $13,000 \mathrm{Da}$, some of which exhibit phospholipase activity (14).

In the present study, harm to the gastrocnemius muscle of Wistar rats injected (IM) with $B$. neuwiedi venom at a dose of $40 \mu \mathrm{g} / \mathrm{mL}$ was observed at all moments except at 7 days. Initially, there were injuries with intensive hemorrhaging, vascular congestion as well as muscle fibers in various stages of degeneration, including condensed myofibrils, delta lesions, fibers with clear areas in the cytoplasm and vacuolized fibers (16). The hemorrhagic condition developed after envenomation triggered muscle injury by means of the action of hemorrhagic toxins (10), which cause damage to the walls of small blood vessels (20). Moreover, the hemorrhagic action of the envenomation process is closely associated with the metalloprotease action in the vascular endothelium, causing disturbances that could lead to cellular lysis.

The myonecrosis may also result from direct action of myotoxins on the muscles fibers or indirect action of hemorrhagic metalloproteases (13). This indirect influence is caused by hemorrhaging, which provokes vascular damage, leading to depletion of the blood that supplies the muscle cells and, in turn, inducing ischemia. The direct action is the effect of myotoxic phospholipases A2 (PLA2s) on plasma membranes of muscle cells, thereby affecting membrane integrity and inducing a rapid increase in plasma CK levels (21).

In the experimental animals, neutrophils had proliferated throughout the entire injured area. At 24 hours following the venom injection, the region of damaged muscle tissue exhibited signs of more accentuated necrosis, presenting the same alterations as observed at three hours following envenomation $(16,17)$.

At three days, the changes in both the venom-only group and the venom-plus-laser group were very similar with regard to the hemorrhagic process and necrosis of the muscle fibers. However, comparing the data on the animals submitted to GaAIAs laser irradiation and the group injected with venom alone, there was a perceptible improvement in the inflammatory and hemorrhagic process at seven days. The 
molecular basis that may explain the effectiveness of laser therapy in the repair of muscle tissue remains unclear.

Karu (14) found that the irradiation of isolated mitochondria induces positive changes to cell homeostasis and suggests that components of the respiratory chain (cytochromes, flavins and dehydrogenase) are capable of absorbing light at a particular wavelength. This absorption is thought to result in an increase of ATP synthesis, thereby affecting hydrogen-ion levels in the cells and activating the ionic gradient (sodium, potassium, calcium). Cytochrome-c-oxidase (terminal enzyme of the respiratory chain) is a photoreceptor in the red band, which is the wavelength used in the present study. The absorption of light by this enzyme is thought to accelerate the transportation of electrons in the respiratory chain, which leads to an increase in the transmembrane electrical potential of the mitochondria, thereby activating ATP synthesis and, consequently, cell metabolism (15).

\section{CONCLUSION}

Through morphological and biochemical analysis, we found that the GaAIAs laser efficiently reduced myonecrosis caused by $B$. neuwiedi venom in the gastrocnemius muscle of Wistar rats.

\section{REFERENCES}

1 ABREU VA., DAL BELO CA., HERNANDES-OLIVEIRA SS., BORJA-OLIVEIRA CR., HYSLOP S., FURTADO MDE F., RODRIGUES-SIMIONI L. Neuromuscular and phospholipase activities of venoms from three subspecies of Bothrops neuwiedi $(B$. n. goyazensis, B. n. paranaensis and B. n. diporus). Comp. Biochem. Physiol. A. Mol. Integr. Physiol., 2007, 148, 142-9.

2 AL-WATBAN FA., ZHANG XY., ANDRES BL. Low-level laser therapy enhances wound healing in diabetic rats: a comparison of different lasers. Photomed. Laser Surg., 2007, 25, 72-7.

3 AMARAL AC., PARIZOTTO NA., SALVINI TF. Dose-dependency of low-energy HeNe laser effect in regeneration of skeletal muscle in mice. Lasers Med. Sci., 2001, 16, 44-51.

4 AVNI D., LEVKOVITZ S., MALTZ L., ORON U. Protection of skeletal muscles from ischemic injury: low-level laser therapy increases antioxidant activity. Photomed. Laser Surg., 2005, 23, 273-7. 
5 BARBOSA AM., VILLAVERDE AB., GUIMARAES-SOUZA L., RIBEIRO W., COGO JC., ZAMUNER SR. Effect of low-level laser therapy in the inflammatory response induced by Bothrops jararacussu snake venom. Toxicon, 2008, 51, 123644.

6 CARRINHO PM., RENNO AC., KOEKE P., SALATE AC., PARIZOTTO NA., VIDAL BC. Comparative study using 685-nm and 830-nm lasers in the tissue repair of tenotomized tendons in the mouse. Photomed. Laser Surg., 2006, 24, 754-8.

7 CARVALHO PT., MAZZER N., DOS REIS FA., BELCHIOR AC., SILVA IS. Analysis of the influence of low-power HeNe laser on the healing of skin wounds in diabetic and non-diabetic rats. Acta Cir. Bras., 2006, 21, 177-83.

8 CORAZZA AV., JORGE J., KURACHI C., BAGNATO VS. Photobiomodulation on the angiogenesis of skin wounds in rats using different light sources. Photomed. Laser Surg., 2007, 25, 102-6.

9 DIAZ C., VALVERDE L., BRENES O., RUCAVADO A., GUTIERREZ JM. Characterization of events associated with apoptosis/anoikis induced by snake venom metalloproteinase BaP1 on human endothelial cells. J. Cell. Biochem., 2005, 94, 520-8.

10 DOURADO DM., FAVERO S., BARANAUSKAS V., DA CRUZ-HÖFLING MA. Effects of the Ga-As laser irradiation on myonecrosis caused by Bothrops moojeni snake venom. Lasers Surg. Med., 2003, 33, 352-7.

11 ELWAKIL TF. An in-vivo experimental evaluation of $\mathrm{He}-\mathrm{Ne}$ laser photostimulation in healing Achilles tendons. Lasers Med. Sci., 2007, 22, 53-9.

12 GUTIERREZ JM., LOMONTE B. Phospholipase A2 myotoxins from Bothrops snake venoms. Toxicon, 1995, 33, 1405-24.

13 HOMMA M., TU AT. Morphology of local tissue damage in experimental snake envenomation. Br. J. Exp. Pathol., 1971, 52, 538-42.

14 KARU TI. Molecular mechanism of the therapeutic effect of low-intensity laser radiation. Lasers Life Sci., 1988, 2, 53-74.

15 KINI RM., EVANS HJ. A model to explain the pharmacological effects of snake venom phospholipases A2. Toxicon, 1989, 27, 613-35.

16 LIRA MS., FURTADO MF., MARTINS LMP., LOPES-FERREIRA M., SANTORO ML., BARBARO KC. Enzymatic and immunochemical characterization of Bothrops insularis venom and its neutralization by polyspecific Bothrops antivenom. Toxicon, 2007, 49, 982-94. 
17 LOMONTE B., MORENO E., TARKOWSKI A., HANSON LA., MACCARANA M. Neutralizing interaction between heparins and myotoxin II, a lysine 49 phospholipase A2 from Bothrops asper snake venom. Identification of a heparin-binding and cytolytic toxin region by the use of synthetic peptides and molecular modeling. $J$. Biol. Chem., 1994, 269, 29867-73.

18 LOPES-MARTINS RA., MARCOS RL., LEONARDO PS., PRIANTI JR AC., MUSCARA MN., AIMBIRE F., FRIGO L., IVERSEN VV., BJORDAL JM. Effect of low-level laser (Ga-Al-As $655 \mathrm{~nm}$ ) on skeletal muscle fatigue induced by electrical stimulation in rats. J. Appl. Physiol., 2006, 101, 283-8.

19 MANTEIFEL VM., KARU TI. Structure of mitochondria and activity of their respiratory chain in successive generations of yeast cells exposed to He- $\mathrm{Ne}$ laser light. Izv. Akad. Nauk. Ser. Biol., 2005, 32, 672-83.

20 MEDRADO AR., PUGLIESE LS., REIS SR., ANDRADE ZA. Influence of low level laser therapy on wound healing and its biological action upon myofibroblasts. Lasers Surg. Med., 2003, 32, 239-44.

21 MERLI LA., SANTOS MT., GENOVESE WJ., FALOPPA F. Effect of low-intensity laser irradiation on the process of bone repair. Photomed. Laser Surg., 2005, 23, 212-5.

22 MORITA, T. Use of snake venom inhibitors in studies of the function and tertiary structure of coagulation factors. Int. J. Hematol., 2004, 79, 123-9.

23 NONAKA PN., AMORIM CF., PANEQUE PERES AC., E SILVA CA., ZAMUNER SR., RIBEIRO W., COGO JC., VIEIRA RP., DOLHNIKOFF M., DE OLIVEIRA LV. Pulmonary mechanic and lung histology injury induced by Crotalus durissus terrificus snake venom. Toxicon, 2008, 51, 1158-66.

24 RIBEIRO LA. Epidemiology of ophidic accidents. Mem. Inst. Butantan, 1990, 52, 15-16.

25 SAlATE AC., BARBOSA G., GASPAR P., KOEKE PU., PARIZOTTO NA., BENZE BG., FOSCHIANI D. Effect of In-Ga-Al-P diode laser irradiation on angiogenesis in partial ruptures of Achilles tendon in rats. Photomed. Laser Surg., 2005, 23, 470-5.

26 SALTMARCHE AE. Low level laser therapy for healing acute and chronic wounds - the extendicare experience. Int. Wound J., 2008, 5, 351-60. 